\title{
Presenteeism: Are We Hurting the Patients We are Trying to Help?
}

\author{
Michael Landry, MD, MSc ${ }^{1,2}$ and Chad Miller, $\mathrm{MD}^{1}$ \\ 'Department of General Internal Medicine and Geriatrics, Tulane University School of Medicine, New Orleans, LA, USA; ${ }^{2}$ Section of General \\ Internal Medicine, Southeast Louisiana Veterans Healthcare System, New Orleans, LA, USA.
}

J Gen Intern Med 25(11):1142-3

DOI: $10.1007 / \mathrm{s} 11606-010-1487-6$

(c) Society of General Internal Medicine 2010

$\mathrm{M}$ any physicians remember the Hippocratic Oath (or a modern variant) as one of the milestones in their professional development. In professing the Hippocratic Oath, physicians promise to "abstain from doing harm". Thomas Syndeham (1624-1689) has been identified as the originator of the Latin phrase primum non nocere translated as "first do no

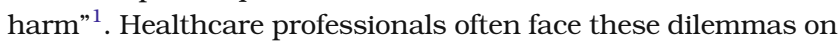
a daily basis either consciously or unconsciously.

While business and industry have long recognized the costs of employee illnesses, the dominant focus has usually been on absenteeism and the resulting costs. However, many times, employees report to work for various reasons even in the face of serious illness. Presenteeism has been defined as "the problem of workers being on the job, but because of illness, not fully functioning". ${ }^{2}$ The cost of lost employee productivity due to presenteeism is estimated to exceed $\$ 150$ billion dollars in the United States alone. ${ }^{2}$ Chronic illnesses dominate the presenteeism literature with little focus on acute illness and communicable diseases. In the healthcare sector, few studies have addressed the question: How effective are healthcare professionals when they report to work acutely ill?

In an article published in this issue, Widera and colleagues report an outbreak of norovirus gastroenteritis in a 100-bed long term care facility. In their case report, healthcare professionals returned to work ill despite increasing evidence that their behaviors were perpetuating the outbreak. Decisions were made to circumnavigate policies enacted to curb the transmission of an identified infectious pathogen ${ }^{3}$. Why would healthcare providers report to work ill and risk disease transmission to their patients in light of this information?

The reasons cited by Widera et al. are varied and may depend on where the healthcare worker resides in the chain of command $^{3}$. Nonetheless, meaningful change should begin with the physicians as the standard for other healthcare workers. Encouraging physicians to stay home is difficult because it is in stark contrast with many behaviors taught and adopted since medical school. From day one, physicians that sacrifice for their patients are viewed as role models. The personal sacrifice required of physicians is seen as noble and honorable. For many, it is a "badge of honor" that nearly nothing will come between them and their patients. Diarrhea, a cold or a cough seem like small obstacles to overcome to help our patients, but

Published online August 26, 2010 could this behavior be unethical, if symptoms are caused by communicable diseases?

Nonmaleficence, a core principle of medical ethics, is often summarized by "do no harm." It is the intentional refraining from actions that cause harm. Negligence is a breach of this principle. ${ }^{4}$ It may come from unintentionally but carelessly imposing risks of harm, such as when physicians knowingly present to work with communicable diseases and transmit them to patients.

National healthcare reform has been enacted and new policies are being developed to advance healthcare. But how do we help healthcare professionals "do the right thing"? Ethically, how do we convince healthcare professionals that absenteeism may be more beneficial to their patients than presenteeism? As concerns over pandemic infections rise, patients need to be aware of the potential impact of a "sick" provider on their well being. Healthcare reform needs to allow healthcare professionals to "just stay home". Patients have the right to demand it. Colleagues have a right to expect it. Education needs to focus on the ethical implications of medical decisions that providers may make regarding their own healthcare which imperil the health of their patients. Memories and anecdotal stories exist of colleagues who worked while quite ill. These ill colleagues were avoided by fellow healthcare professionals over the course of that day, while patients were unwittingly exposed to communicable diseases.

But what options do patients have once their healthcare provider enters their room? The provider may not display signs of illness. In addition, patients may not be capable of prematurely ending their clinic visit, hospital admission or surgery. Patients should be able to obtain healthcare in a safe and disease free environment. When patients seek healthcare, they often assume that fellow patients may be sick and seek ways of protecting themselves, but could the one who is supposed to heal them be their bigger concern? For example, studies have shown that healthcare professionals fail to observe prescribed hand-washing recommendations before and after patient encounters ${ }^{5,6}$ and that medical care is fraught with perils like disease carrying neckties ${ }^{7}$ or stethoscopes $^{8}$. Potential disease carrying providers who continue with normal healthcare routines should reflect on the ethical implications of their behavior. These same illnesses would often result in healthcare intervention by the provider if the same scenario were presented to them as a sick patient.

Under the Deficit Reduction Act of 2005 (section 5001 ( )), "conditions that could reasonably have been prevented through the application of evidence based guidelines"9 need to be identified. In July 2008, the Center for Medicare and Medicaid Services identified ten hospital acquired conditions that impact payments in the inpatient setting. Similarly, if healthcare providers report to work ill and their patients suffer 
adverse outcomes from this exposure, should reimbursement be affected? Guidelines can help physicians provide clinical care; but proper judgment may be lacking if providers continue to work ill while informing their patients of a different set of clinical standards.

Widera and colleagues propose several options as starting points for discussion. The first proposal involves liberal paid sick leave to decrease financial pressures for individuals to return to work earlier than expected. Second, they advocate for systematic processes that screen individuals for communicable diseases especially during disease outbreaks. Lastly, adequate staffing must be appropriated to avoid the often incorrect feeling that "my patients are better off with me there sick than if I am not there" mentality ${ }^{3}$.

Interestingly, there may be an alternative to "just staying home" that many physicians may find more palatable. Technology already exists that could allow physicians to keep working, but removes the risk of infection. Although the trend to treating the "patient as icon" is concerning, this may be an instance where its conscious application is reasonable. Telemedicine by phone, videophone, Internet, or email, may be an acceptable alternative when a face to face visit is not absolutely necessary. It would allow physicians to triage patients for the day and refer those that need further assessments to a colleague. Physicians may not have to "stay home," but could "stay away." This would limit the impact of lost wages for physicians, the guilt of not being able to care for one's own patients and the burden of work passed to busy colleagues. The recent success of the remote ICU could be a model used for scenarios where the doctor is sick and needs to "stay away." Clearly, this measure may not apply to many healthcare workers who must be physically present to perform their jobs, but it sends the appropriate message from physicians that patients should not be exposed to our communicable illnesses. Support of this "remote physician" concept would need healthcare reimbursement reform for these types of activities.
This thought-provoking manuscript reveals that education is necessary as providers must realize that they are not above the system they work in, but rather they are part of the system. If providers report to work ill, patients risk exposure to infectious illnesses. Providers tell patients that it is in the patient's best interest if they stay at home and rest, but these same providers may fail to follow these instructions. Innovations like telemedicine could be utilized when the physician becomes a patient.

Would we as healthcare professionals be better physicians if we were better patients?

\section{Conflict of Interest: None disclosed}

Corresponding Author: Michael Landry, MD, MSc; Department of General Internal Medicine and Geriatrics, Tulane University School of Medicine, 1430 Tulane Avenue SL 16. New Orleans, LA 70112, USA (e-mail: mlandry@tulane.edu).

\section{REFERENCES}

1. Smith, Cedric M. Origin and uses of Primum Non Nocere-Above All, Do No Harm. J Clin Pharmacol. 2005;45:371-7.

2. Hemp P. Presenteeism: at work-but out of it. Harv Bus Rev. 2004;82 (10):49-58. 155

3. Widera E, Chang A, Chen H. Presenteeism: A Public Health Hazard. Journal of General Internal Medicine 2010; xxxxxx

4. Beauchamp T, Childress J. Principles of Biomedical Ethics. 6th ed. New York, NY: Oxford University Press; 2009.

5. Pittet D, Mourouga P, Perneger TV. Compliance with handwashing in a teaching hospital. Ann Intern Med. 1999;130:126-30.

6. Goldman D. System failure versus personal accountability-The case for good handwashing. New England Journal of Medicine 2006: volume 335 (2), 121-123

7. Day, M. Doctors are told to ditch "disease spreading" neckties. British Medical Journal 2006: 332:442

8. Fenelon L, Holcroft L, Waters N. Contamination of stethoscopes with MRSA and current disinfection practices. J. Hosp. Infection. 2009;71 (4):378-8.

9. Center for Medicare and Medicaid Services. www.cms.gov 